\title{
COVID-19 vaccination-associated anti-Jo-1 syndrome
}

\author{
Kushagra Gupta ${ }^{1}$ ID , Gauri Shankar Sharma ${ }^{2}$, Ashok Kumar ${ }^{1}$ \\ ${ }^{1}$ Department of Rheumatology, Fortis Flt. Lt. Rajan Dhall Hospital, New Delhi, India \\ ${ }^{2}$ Department of Critical Care Medicine, Fortis Flt. Lt. Rajan Dhall Hospital, New Delhi, India
}

\begin{abstract}
Post-vaccination inflammatory myositis is a rare but known entity in the literature. We encountered a 46-year-old female patient, who presented with complains of fever, arthralgia, and weakness 1 week after taking the second dose of COVID-19 (Oxford-AstraZeneca) vaccine. On workup the patient had raised inflammatory markers, evidence of myositis on magnetic resonance imaging of thighs, and evidence of interstitial lung disease on high-resolution computed tomography of the chest. The patient was further found to be positive for anti-Jo-1 antibody. The initial treatment was glucocorticosteroids and methotrexate initially. The patient briefly developed pneumocystis pneumonia and recovered. The treatment was switched to mycophenolate mofetil with good response. We presented the first case of anti-Jo-1 syndrome reported following COVID-19 vaccination in the literature. Our aim is to sensitise the clinicians to such rare but occasionally life-threatening complications that may arise in the post-vaccination period.
\end{abstract}

Key words: anti-Jo-1 syndrome, vaccination, COVID-19 vaccine, myositis.

We wish to report a rare case of anti-Jo-1 syndrome, which occurred shortly after COVID-19 vaccination.

A 46-year-old female patient, a homemaker by profession, presented with complaints of low-grade fever and joint pains of 1-week duration. The pain was mainly restricted to the large joints, with no obvious tenderness or swelling on examination. This was associated with generalized weakness and a feeling of fatigue persisting throughout the day. The described patient received a second dose of COVID-19 vaccination (Covishield Oxford-AstraZeneca vaccine manufactured in India) just 1 week prior to the onset of her symptoms. Although no symptoms were observed after the first vaccine dose, this was considered as an adverse event following immunization and was treated with non-steroidal anti-inflammatory drugs. However, the patients' complaints persisted.

Laboratory tests revealed leukocytosis of 14,610 per $\mu \mathrm{l}(4500-11,000$ per $\mu \mathrm{l})$ with a differential of $81 \%$ neutrophils and $12 \%$ lymphocytes. Liver function tests showed elevated aspartate aminotransferase and alanine aminotransferase: 102 and $50 \mathrm{U} / \mathrm{l}(<40 \mathrm{U} / \mathrm{l})$, respectively, raised lactate dehydrogenase: $768 \mathrm{U} / \mathrm{l}$ (140-280 U/l); also, inflammatory markers such as erythrocyte sedimentation rate: $48 \mathrm{~mm} /$ hour ( $<30 \mathrm{~mm} /$ hour) and C-reactive protein: $76 \mathrm{mg} / \mathrm{l}(<10 \mathrm{mg} / \mathrm{l})$ were elevated. Other parameters including haemoglobin, renal function test, and thyroid function assessment were within normal range.

In view of persisting fever and the ongoing COVID-19 pandemic, the patient was evaluated for the possibility of active SARS-CoV-2 infection by real-time polymerase chain reaction (RT-PCR), which was negative. Lung imaging using high-resolution computed tomography (HRCT) revealed interstitial reticulations with septal thickening in peripheral distribution involving bilateral basal regions, suggestive of early interstitial lung disease. This is shown in Figure $1 \mathrm{~A}$.

Immunological tests showed rheumatoid factor, anticitrullinated cyclic protein antibodies and antinuclear antibodies to be negative. The patient was referred to our rheumatology clinic for further evaluation.

On physical examination, there was tenderness of biceps brachii and thigh muscles with a power of 4/5 in all proximal muscle groups, which was assessed by the Medical Research Council Scale of muscle strength test-

\section{Address for correspondence:}

Kushagra Gupta, Department of Rheumatology, Fortis Flt Lt Rajan Dhall Hospital, Aruna Asif Ali Marg, Vasant Kunj, New Delhi-110070, India, e-mail: kushagrahsr@gmail.com

Submitted: 06.11.2021; Accepted: 29.11.2021 

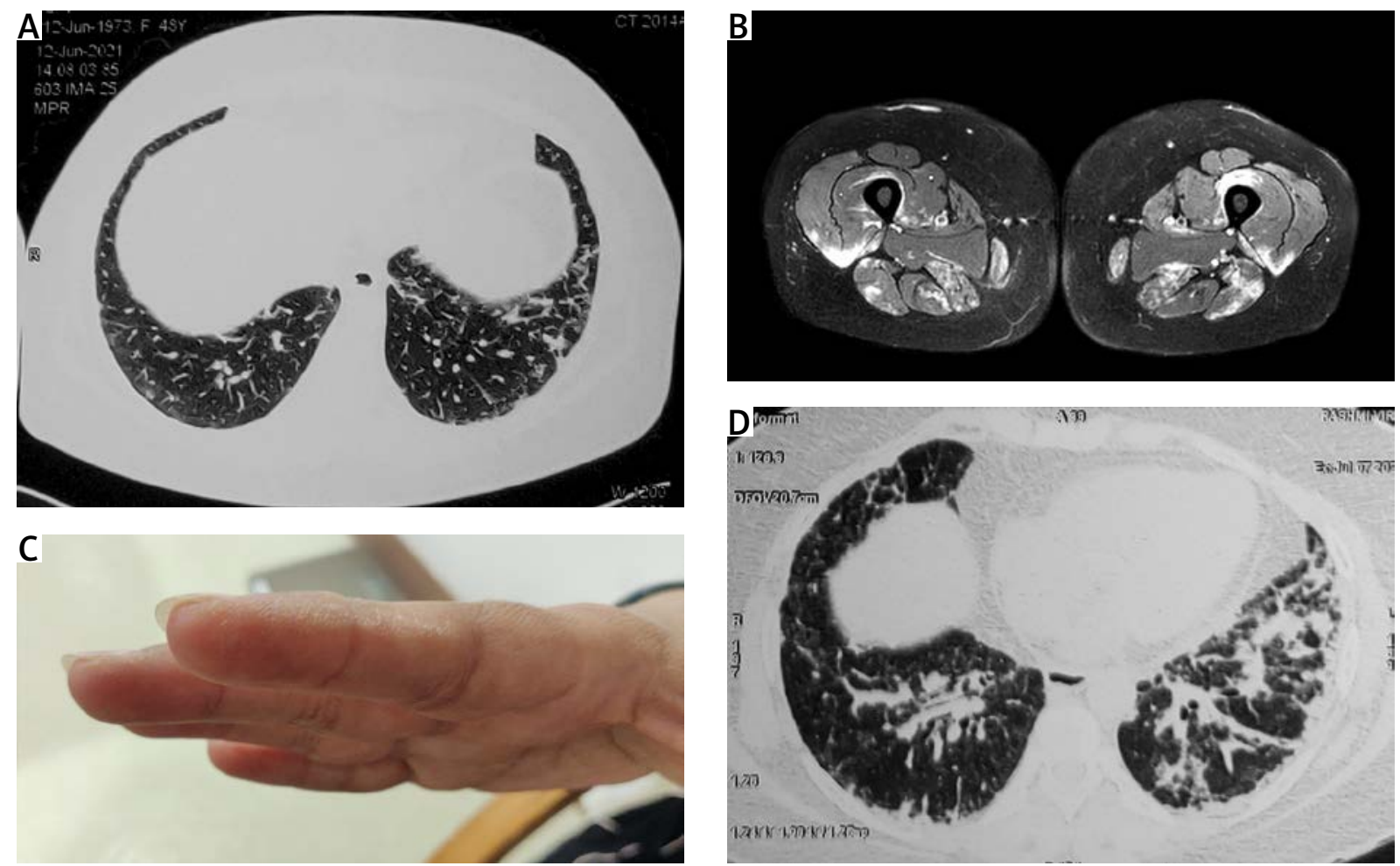

Fig. 1. Images and a clinical photograph: HRCT chest showing interstitial reticulations and septal thickening suggestive of early ILD (A), MRI of both thighs showing increased uptake in bilateral muscle groups of thighs suggestive of active myositis (B), hyperkeratotic eruptions on the radial aspect of index finger suggestive of 'Mechanic's hands' (C), repeat HRCT chest showing increased infiltrates in lower lobes after initiation of glucocorticoids and methotrexate (D).

ing using a score of 0 to 5 . The patient was also found to have mechanic's hands with a corresponding image presented in Figure $1 \mathrm{~B}$.

Electromyography showed myopathic changes, suggestive of primary muscle disease. Magnetic resonance imaging of the muscles showed increased uptake in bilateral muscle groups suggestive of myositis, which is presented in Figure $1 \mathrm{C}$. Creatine phosphokinase levels were $1560 \mathrm{U} / \mathrm{l}$, and the myositis profile was positive for anti-Jo-1 and anti-Ro-52 antibodies.

The patient's medical history was re-examined, and no confirmation for contact with any toxins was found. The presented symptoms had a temporal relationship with COVID vaccination, and a diagnosis of COVID vaccine associated anti-Jo-1 syndrome was made. We decided to start treatment with prednisolone $1 \mathrm{mg} / \mathrm{kg}$ along with methotrexate $15 \mathrm{mg} /$ week. After 3 weeks, the patient was admitted with worsening breathing problems. Tachypnoea and a low oxygen saturation of $90 \%$ was found. Repeat HRCT showed increased interstitial infiltrates (Fig. 1 D), and a possibility of methotrexate pneumonitis, after exclusion of any infection, was considered.

Methotrexate was withdrawn and the dose of prednisolone was reduced; because there was a fear of an underlying source of infection, antibiotics were started. The patient also required oxygen treatment. The bronchoscopy evaluation was performed with bronchoalveolar lavage examination, and the result was positive for pneumocystis jirovecii by PCR analysis. All other cultures were sterile. The patient was started on treatment with co-trimoxazole (sulfamethoxazole and trimethoprim) and responded well to the therapy. The patient was gradually weaned off oxygen support. On follow-up, mycophenolate mofetil was added to the treatment along with glucocorticosteroids. At present, the patient feels well and does not require any treatment modification.

A short discussion of the case is warranted because we know that vaccines are among the environmental factors that have been proposed as triggering factors in the pathogenesis of inflammatory myopathies. Various components of the vaccine, including the antigen, adjuvant, preservatives, and stabilizers, have been associated with autoimmunity [1]. Adjuvants, in particular, are substances that are incorporated in the vaccines to boost immune response against a desired antigen. Similarly, adjuvants can also boost a previously circulating self-antigen in a genetically susceptible individual, which was otherwise kept in check by the immune sys- 
tem. This can lead to breakdown of the immune tolerance barrier and precipitate autoimmunity [1]

Autoimmune syndromes that are induced following exposure to adjuvants have been termed autoimmune/ inflammatory syndrome induced by adjuvants (ASIA) [2]. There have been several case reports in the literature describing inflammatory myopathies occurring after immunization with vaccines like MMR vaccine, influenza, Bacillus Calmette-Guerin, and human papilloma virus vaccine [3].

In fact, there are a vast array of rheumatological conditions reported following vaccination ranging from arthritis, myelitis, and vasculitis to fibromyalgia [1, 4] However, these conditions are so rare and sporadic that it is difficult for epidemiological studies to establish an association between them and autoimmunity in general. In our literature search, we encountered just 1 case report of anti-synthetase syndrome that occurred following influenza vaccine [5]; however, no such cases have been reported in association with COVID vaccination.

On the basis of the presented case, we wanted to highlight the fact that rheumatologists should be aware of the possibility of autoimmune syndromes that can occur following vaccination. Especially now, with the global program of vaccination against SARS-CoV-2, such cases should be reported and collected to search for regularities that would help to avoid such phenomena and to isolate other potential factors of their occurrence independently of vaccination. Fortunately, such adverse effects are rare, and the usefulness of large-scale vaccinations against COVID-19 is well documented. In our opinion, this case-report should not constitute any reason for discouraging vaccination.

The authors declare no conflict of interest.

\section{References}

1. Guimarães LE, Baker B, Perricone C, Shoenfeld Y. Vaccines, adjuvants and autoimmunity. Pharmacol Res 2015; 100: 190-209, DOI: 10.1016/j.phrs.2015.08.003.

2. Shoenfeld Y, Agmon-Levin N. ASIA - autoimmune/inflammatory syndrome induced by adjuvants. J Autoimmun 2011; 36: 4-8, DOI: 10.1016/j.jaut.2010.07.003.

3. Stübgen JP. A review on the association between inflammatory myopathies and vaccination. Autoimmun Rev 2014; 13: 31-39, DOI: 10.1016/j.autrev.2013.08.005.

4. Maślińska M, Perricone C, Shoenfeld Y. Autoimmune/inflammatory syndrome induced by adjuvants - a new diagnostic problem or the solution of a diagnostic riddle. Reumatologia 2013; 51: 437-444, DOI:10.5114/reum.2013.39662.

5. Philip C, Kabani N, Keith R, et al. Antisynthetase Syndrome Induced by influenza vaccine: a unique case of ASIA Syndrome. J Clin Rheumatol 2019, DOI: 10.1097/RHU.0000000000001015 [Online ahead of print]. 